BMJ Open Sport \& Exercise Medicine

\title{
Can foot angle influence the risk of injury to the lower limb joints during a field hockey hit?
}

\author{
Frances E Feeley, ${ }^{1}$ Graham P Arnold,${ }^{1}$ Sadiq Nasir, ${ }^{1}$ Weijie W Wang, ${ }^{1}$ \\ Rami Abboud (10 ${ }^{2}$
}

To cite: Feeley FE, Arnold GP, Nasir S, et al. Can foot angle influence the risk of injury to the lower limb joints during a field hockey hit? BMJ Open Sport \& Exercise Medicine 2019;5:e000568. doi:10.1136/ bmjsem-2019-000568

Accepted 7 0ctober 2019
Check for updates

(c) Author(s) (or their employer(s)) 2019. Re-use permitted under CC BY-NC. No commercial re-use. See rights and permissions. Published by BMJ.

${ }^{1}$ Institute of Motion Analysis \& Research (IMAR), Orthopaedic \& Trauma Surgery, University of Dundee, Dundee, UK ${ }^{2}$ Faculty of Engineering, University of Balamand, Al Koura Campus, Balamand, Lebanon

Correspondence to Professor Rami Abboud; rjabboud@balamand.edu.lb

Dr Graham P Arnold; g.p.arnold@dundee.ac.uk

\section{ABSTRACT}

Objectives The lower limb is widely reported as the most commonly injured body part in the field of hockey, more specifically lateral ankle sprains and internal knee injury. Despite this, there remains limited understanding of how the biomechanics of the sport could be adapted to minimise injury. The aim of this study was to propose a foot position during the hockey hit that results in the smallest joint angles and moments, from a total of four different foot positions: $0^{\circ}, 30^{\circ}, 60^{\circ}$ and $90^{\circ}$, which may correlate to injury risk.

Method Eighteen players from the local University Ladies Hockey Club participated in this study. Each player was required to perform a hit with their lead foot in four different positions: $0^{\circ}, 30^{\circ}, 60^{\circ}$ and $90^{\circ}$, where $0^{\circ}$ was a lead foot position perpendicular to the direction of motion of the ball. Angles and moments were calculated with the Vicon system using force plates and motion analysis.

Results Significant differences $(p<0.05)$ were found between the angles and moments of the four foot positions tested, indicating that foot angle can influence the degree of angulation, and moments, produced in the lower limb joints during the hockey hit.

Conclusion There is a relationship between lead foot position and the angles and moments produced in the lower limb joints during the hockey hit, and this may correlate with injury risk.

\section{INTRODUCTION}

Field hockey is a fast-paced stick and ball sport played in 132 countries worldwide. ${ }^{1}$ Players must withstand forces generated from fast running and sharp turns while also using their upper body to control and strike the ball.

Although contact injuries from the stick and ball are more common and can have serious consequences, non-contact mechanisms are significant, particularly among female players. ${ }^{2}$ The lower limb is of particular interest; Barboza et a $\vec{l}$ carried out a systematic review of injury data and found that this was the area of the body most commonly injured during hockey, more specifically the knee and ankle with the literature vague on whether

\section{What are the new findings?}

Lead foot position influences the angles and moments produced in the lower limb joints during the hockey hit.

- Overall, a lead foot position in line with the rest of the body whilst performing the hockey hit, defined as $30^{\circ}$ in the present study, produced the lowest angles and moments in the most significant planes of motion.

- Foot position may correlate with injury risk to the lower limb during the hockey hit.

the injuries occur through hitting or running. The complex cutting manoeuvres and highpower swing motions required to distribute the ball create a high risk of overuse injury, particularly to the ligaments of the knee and lateral ankle. ${ }^{4}$ However, limited literature exists on the biomechanics of the sport and how this relates to non-contact injury mechanisms.

Degree of angulation and magnitude of moments around a joint are factors known to correlate with the risk of injury, as they play a key role in the biomechanics of the joint. ${ }^{5-7}$ Since the lower limb joints allow limited degrees of angulation, particularly in the coronal and transverse planes, ${ }^{8}$ a foot position that results in angulation of the foot close to its maximum angle, in the respected plane of motion, will increase the risk of injury. Furthermore, there are a number of factors that influence how the magnitude of a force will affect the joint, such as the strength of surrounding muscles. Therefore, there is not a particular magnitude of moment that can be stated as the threshold for injury, making it difficult to quantify the risk of injury. However, through comparison of the four positions against one another, the one that produced the smallest moments the most often, and largest moments the least often, could be said to carry the smallest risk of injury. 


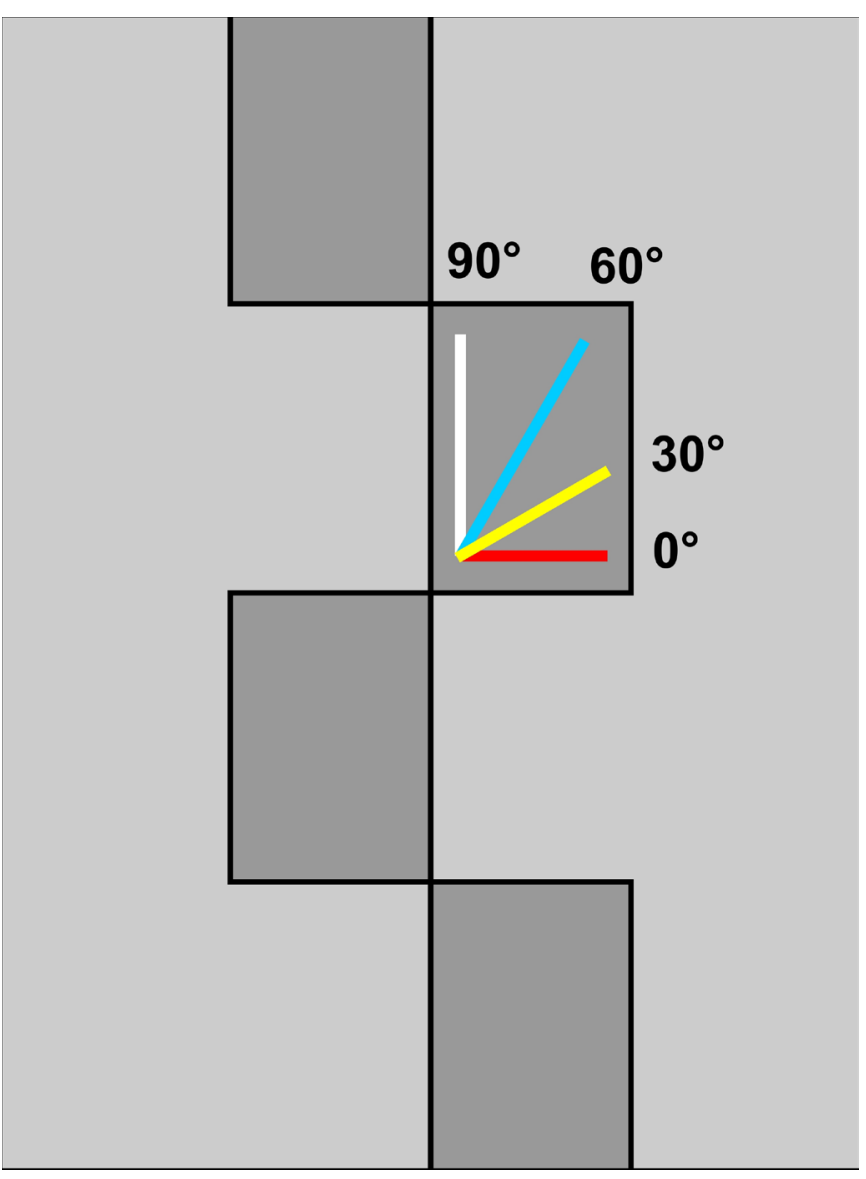

Figure 1 Foot positions. ${ }^{11}$

There were four foot positions tested in the present study: $0^{\circ}, 30^{\circ}, 60^{\circ}$ and $90^{\circ}$, relative to the axes of the force plate used to gather motion analysis data. In hockey, a side on stance is common, with the front foot placed at a diagonal to the line of movement of the ball. In this position, the front-foot faces in a similar direction to the rest of the body, with minimal rotation of the ankle joint relative to the body. In the present study, this foot position was defined as $30^{\circ}$. In order to gather motion analysis data with both a smaller and larger degree of angulation at the ankle, a further three foot positions, defined as $0^{\circ}$, $60^{\circ}$ and $90^{\circ}$ were also tested.

A foot position of $90^{\circ}$ was the highest degree included because this results in the foot pointing in the direction of movement. A fourth angle of $60^{\circ}$ was included for a more thorough comparison of foot positions between the two extremes of $0^{\circ}$ and $90^{\circ}$.

The effect of foot position during a drag flick, a type of stroke performed in hockey when shooting at goal, was investigated by Wild et $a l^{9}{ }^{9}$ The authors proposed that an externally rotated lead foot position during this stroke increases the force at the ankle joint. The hit, which was analysed in the present study, is relevant to a wider range of hockey players than the drag flick, as it is used in all aspects of the game. Therefore, understanding the biomechanics of this stroke is highly relevant.
It appears that adaptation of foot orientation is possible through appropriate training. A recent study involving a neuromuscular training programme for hockey players classed as having unstable ankles resulted in a positive effect on the participants' ankle positioning. ${ }^{10}$

This study aimed to propose a lead foot position during the hockey hit that results in the smallest joint angles and moments, from a total of four different foot positions: $0^{\circ}, 30^{\circ}, 60^{\circ}$ and $90^{\circ}$. The null hypothesis of this study was that no relationship exists between lead foot position and the angles and moments produced during a hockey hit.

\section{MATERIALS AND METHODS Patient and public involvement}

Twenty female hockey players were recruited from the local University Ladies Hockey Club. Volunteers were recruited through a poster being displayed in the hockey club and were given a participant information sheet prior to the study commencing. Participants were required to have played a minimum of one season of competitive hockey and have no significant injuries that precluded them from playing the year before the study was conducted.

\section{Procedure}

Motion analysis data were collected using the Vicon Nexus system V.2.6.1 (using 14 MXF40 cameras and 4 AMTI force plates BP600400). Coloured tape was used to mark the four foot positions on one of the four force plates, as shown in figure 1.

Before collecting data, each participant was provided with a standardised pair of hockey shoes in the appropriate size to minimise any variations that could be attributed to footwear. Anthropometric data were recorded. Sixteen retroreflective markers were then attached at the following bony landmarks: anterior superior iliac spine, sacral dimple, medial and lateral femoral epicondyle, medial and lateral malleoli, posterior calcanei and between the first and second metatarsal heads. A further four wand markers were placed on each lateral thigh and calf (figure 2). Following calibration of the laboratory, participants were provided with a ball and a standard hockey stick that matched their height and asked to practice performing the hit until the participant and lead investigator agreed that they were familiar with the experimental setup. The hit was then performed while stepping onto the force plate. The trial was considered successful if the motion was performed correctly, with their whole foot on the force plate, and at the required angle. Data were collected until five successful trials at all four foot positions were recorded, from each participant.

\section{Data analysis}

Vicon software was used to label successful trials. Trials were disregarded if any of the markers were missing, if the foot position was not at the required angle, or if the foot was not completely within the boundary of the force 

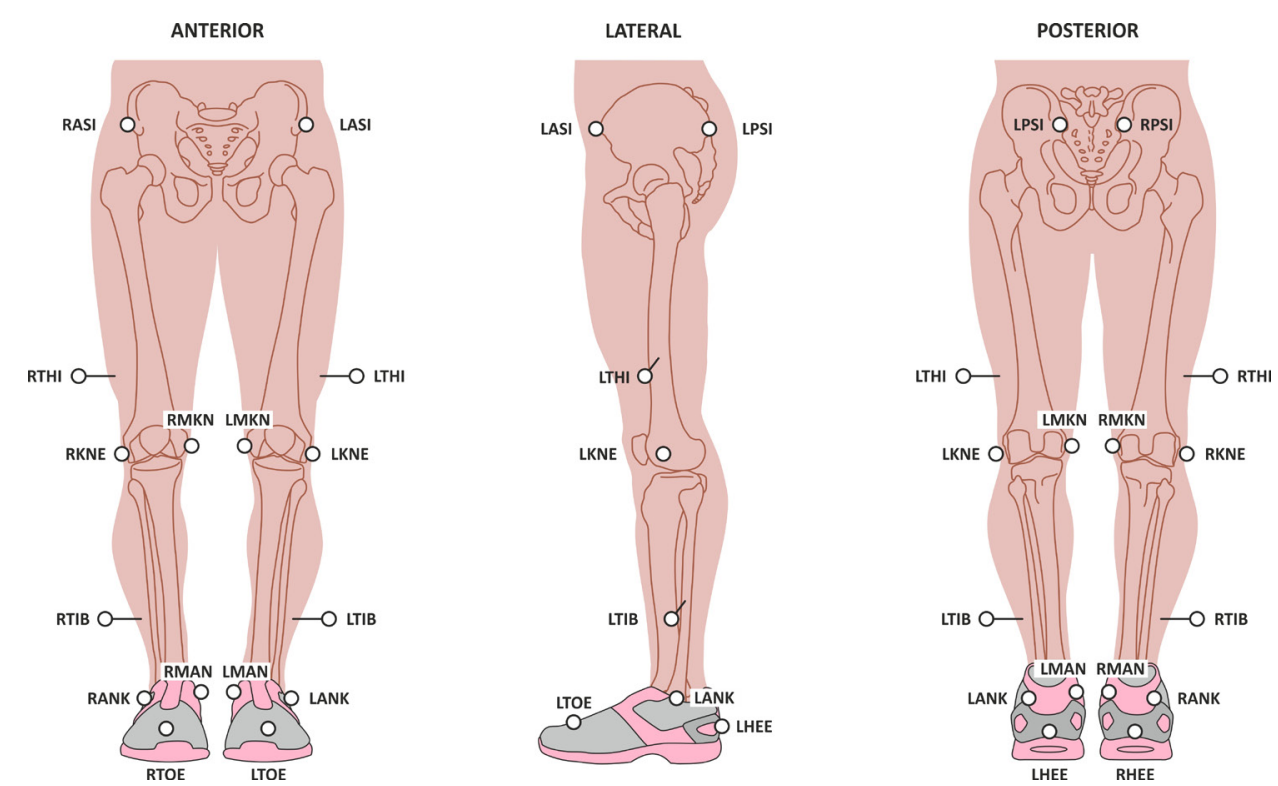

Figure 2 Marker placement. ${ }^{12}$ LANK, left ankle (lateral); LASI, left asis; LHEE, left heel; LKNE, left knee (lateral); LMANK, left ankle (medial); LMKNE, left knee (medial); LPSI, left psis; LTHI, left thigh; LTIB, left tibia; LTOE, left toe; RANK, right ankle (lateral); RASI, right asis; RHEE, right heel; RKNE, right knee (lateral); RMANK; right ankle (medial); RMKNE, right ankle (medial); $\mathrm{RPSI}$, right psis; $\mathrm{RTHI}$, right thigh; RTIB, right tibia; $\mathrm{RTOE}$, right toe;

plate. This was the case for two participants, so data from eighteen participants was analysed.

\section{Statistical analysis}

The SPSS system V.22 was used to analyse the data. Analysis and comparison between foot positions was carried out using the general linear model and pairwise comparisons. Four groups were formed using information from all 18 participants at each foot position. A significant difference was reported if the $p$ value was $<0.05$.

\section{RESULTS}

Of the 18 participants whose data were analysed, the mean age was 20 years (SD 1.0); the mean height was $167 \mathrm{~cm}$ (SD 5.2) and the mean mass was $64.2 \mathrm{~kg}$ (SD $5.7)$.

Graphs were created to clearly display the trends of angles and moments between the foot positions.

Due to lateral ankle sprains and internal knee injury being the most common injuries in hockey, particular focus was paid to the planes of motion in which these could occur. Statistically significant differences $(p<0.05)$ were found between the angles and moments of all four foot positions tested.

The effect size, CIs and $\mathrm{p}$ values for all comparisons made are shown in tables 1 and 2, for ankle and knee data, respectively.

\section{Ankle}

Plantarflexion

Angles

The maximum plantarflexion angles were lowest at a foot position of $30^{\circ}$ with a mean of $15.3^{\circ}$, and highest at $60^{\circ}$ with a mean of $20^{\circ}$. The only significant difference was between $30^{\circ}$ and $60^{\circ}(\mathrm{p}<0.05)$.

\section{Moments}

Angle $0^{\circ}$ produced the lowest maximum plantarflexion moment of the four foot positions $(121 \mathrm{Nmm} / \mathrm{kg})$, and this degree was significantly different $(\mathrm{p}<0.001)$ from the other three, of which $30^{\circ}$ produced the highest result $(273 \mathrm{Nmm} / \mathrm{kg})$. There were no significant differences between $30^{\circ}, 60^{\circ}$ or $90^{\circ}$.

\section{Inversion}

\section{Angles}

As seen in figure 3 , the two foot positions that produced the lowest maximum inversion angles were $0^{\circ}$ and $30^{\circ}$, between which no significant difference was found. Foot positions of $90^{\circ}$ and $60^{\circ}$ produce the highest inversion angles and no significant difference was found between them. However, significant differences were found between $0^{\circ}$ and both $60^{\circ}$ and $90^{\circ}$, and $30^{\circ}$ and both $60^{\circ}$ and $90^{\circ}(\mathrm{p}<0.001)$.

\section{Moments}

Figure 3 shows that $30^{\circ}$ and $60^{\circ}$ produced the smallest inversion moments of the four positions and were not significantly different from each other. The maximum moment at $90^{\circ}(113 \mathrm{Nmm} / \mathrm{kg})$, which was the highest of the four positions, was significantly different from $0^{\circ}(\mathrm{p}<0.05)$ and of greater significant difference from $30^{\circ}$ and $60^{\circ}(\mathrm{p}<0.001)$. No significant differences were found between $0^{\circ}, 30^{\circ}$ and $60^{\circ}$. 


\section{Open access}

Table 1 Ankle data

\begin{tabular}{|c|c|c|c|c|c|c|c|}
\hline \multirow{2}{*}{ Event type } & & & \multirow[b]{2}{*}{ Mean difference } & \multirow[b]{2}{*}{ SE } & \multirow[b]{2}{*}{$P$ value } & \multicolumn{2}{|c|}{ 95\% Cl for difference } \\
\hline & & & & & & Lower bound & Upper bound \\
\hline \multirow{12}{*}{$\begin{array}{l}\text { Plantarflexion } \\
\text { angles (degrees) }\end{array}$} & \multirow[t]{3}{*}{0} & 30 & 1.381 & 2.162 & 0.526 & 5.718 & 2.956 \\
\hline & & 60 & 3.288 & 1.970 & 0.101 & 0.663 & 7.238 \\
\hline & & 90 & 0.919 & 1.736 & 0.599 & 2.563 & 4.401 \\
\hline & \multirow[t]{3}{*}{30} & 0 & 1.381 & 2.162 & 0.526 & 2.956 & 5.718 \\
\hline & & 60 & $4.669^{*}$ & 1.607 & 0.005 & 1.445 & 7.893 \\
\hline & & 90 & 2.300 & 1.647 & 0.168 & 1.003 & 5.603 \\
\hline & \multirow[t]{3}{*}{60} & 0 & 3.288 & 1.970 & 0.101 & 7.238 & 0.663 \\
\hline & & 30 & $4.669^{*}$ & 1.607 & 0.005 & 7.893 & 1.445 \\
\hline & & 90 & 2.369 & 1.364 & 0.088 & 5.105 & 0.367 \\
\hline & \multirow[t]{3}{*}{90} & 0 & 0.919 & 1.736 & 0.599 & 4.401 & 2.563 \\
\hline & & 30 & 2.300 & 1.647 & 0.168 & 5.603 & 1.003 \\
\hline & & 60 & 2.369 & 1.364 & 0.088 & 0.367 & 5.105 \\
\hline \multirow{12}{*}{$\begin{array}{l}\text { Plantarflexion } \\
\text { moments }(\mathrm{Nmm} / \mathbf{k g})\end{array}$} & \multirow[t]{3}{*}{0} & 30 & $151.735^{\star}$ & 27.226 & 0.000 & 97.127 & 206.344 \\
\hline & & 60 & $148.726^{\star}$ & 28.901 & 0.000 & 90.757 & 206.694 \\
\hline & & 90 & $133.799^{\star}$ & 29.298 & 0.000 & 75.035 & 192.562 \\
\hline & \multirow[t]{3}{*}{30} & 0 & $151.735^{\star}$ & 27.226 & 0.000 & 206.344 & 97.127 \\
\hline & & 60 & 3.009 & 18.310 & 0.870 & 39.735 & 33.716 \\
\hline & & 90 & 17.936 & 13.642 & 0.194 & 45.299 & 9.427 \\
\hline & \multirow[t]{3}{*}{60} & 0 & $148.726^{\star}$ & 28.901 & 0.000 & 206.694 & 90.757 \\
\hline & & 30 & 3.009 & 18.310 & 0.870 & 33.716 & 39.735 \\
\hline & & 90 & 14.927 & 14.173 & 0.297 & 43.354 & 13.500 \\
\hline & \multirow[t]{3}{*}{90} & 0 & 133.799* & 29.298 & 0.000 & 192.562 & 75.035 \\
\hline & & 30 & 17.936 & 13.642 & 0.194 & 9.427 & 45.299 \\
\hline & & 60 & 14.927 & 14.173 & 0.297 & 13.500 & 43.354 \\
\hline \multirow{12}{*}{$\begin{array}{l}\text { Inversion angles } \\
\text { (degrees) }\end{array}$} & \multirow[t]{3}{*}{0} & 30 & 0.329 & 0.665 & 0.623 & 1.663 & 1.006 \\
\hline & & 60 & $3.260^{\star}$ & 0.609 & 0.000 & 4.480 & 2.039 \\
\hline & & 90 & $3.718^{*}$ & 0.621 & 0.000 & 4.964 & 2.472 \\
\hline & \multirow[t]{3}{*}{30} & 0 & 0.329 & 0.665 & 0.623 & 1.006 & 1.663 \\
\hline & & 60 & $2.931^{*}$ & 0.499 & 0.000 & 3.932 & 1.930 \\
\hline & & 90 & $3.389^{*}$ & 0.473 & 0.000 & 4.339 & 2.440 \\
\hline & \multirow[t]{3}{*}{60} & 0 & $3.260^{\star}$ & 0.609 & 0.000 & 2.039 & 4.480 \\
\hline & & 30 & $2.931^{*}$ & 0.499 & 0.000 & 1.930 & 3.932 \\
\hline & & 90 & 0.458 & 0.370 & 0.221 & 1.202 & 0.285 \\
\hline & \multirow[t]{3}{*}{90} & 0 & $3.718^{*}$ & 0.621 & 0.000 & 2.472 & 4.964 \\
\hline & & 30 & $3.389^{*}$ & 0.473 & 0.000 & 2.440 & 4.339 \\
\hline & & 60 & 0.458 & 0.370 & 0.221 & 0.285 & 1.202 \\
\hline
\end{tabular}

Continued 
Table 1 Continued

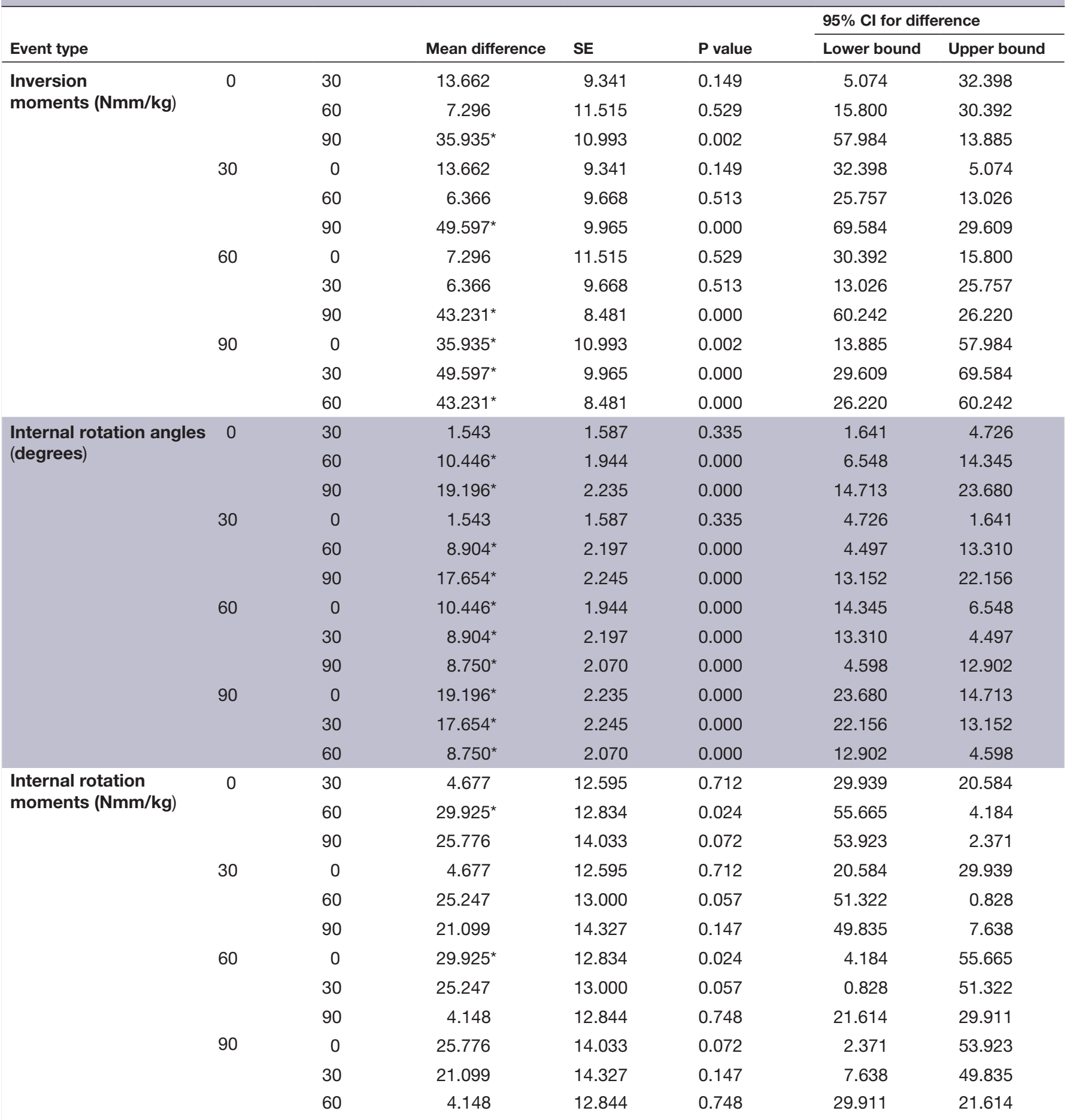

*Highlights data: $p<0.05$.

Internal rotation

\section{Angles}

In the transverse plane, internal rotation angles decreased from a foot position of $0^{\circ}$ to that of $90^{\circ}$, with mean angles of $48.7^{\circ}$ and $29.5^{\circ}$, respectively. Significant differences were found between all four foot angles $(p<0.001)$ except between $0^{\circ}$ and $30^{\circ}$, which produced the highest degrees of internal rotation and were not statistically significant from each other.

\section{Moments}

Furthermore, $60^{\circ}$ produced the highest internal rotation moments $(295 \mathrm{Nmm} / \mathrm{kg})$ and this result was significantly different from that of $0^{\circ}(\mathrm{p}<0.05)$, which produced the lowest $(265 \mathrm{Nmm} / \mathrm{kg})$. However, there are no significant differences between any of the other positions. 


\section{Open access}

Table 2 Knee data

\begin{tabular}{|c|c|c|c|c|c|c|c|}
\hline \multirow{2}{*}{ Event type } & & & \multirow{2}{*}{ Mean difference } & \multirow[b]{2}{*}{ SE } & \multirow[b]{2}{*}{$P$ value } & \multicolumn{2}{|c|}{$95 \% \mathrm{Cl}$ for difference } \\
\hline & & & & & & Lower bound & Upper bound \\
\hline \multirow{12}{*}{$\begin{array}{l}\text { Flexion angles } \\
\text { (degrees) }\end{array}$} & \multirow[t]{3}{*}{0} & 30 & 0.445 & 1.053 & 0.674 & 2.556 & 1.667 \\
\hline & & 60 & $4.027^{*}$ & 1.124 & 0.001 & 6.282 & 1.772 \\
\hline & & 90 & $6.149^{*}$ & 1.390 & 0.000 & 8.937 & 3.360 \\
\hline & \multirow[t]{3}{*}{30} & 0 & 0.445 & 1.053 & 0.674 & 1.667 & 2.556 \\
\hline & & 60 & $3.582^{*}$ & 1.150 & 0.003 & 5.889 & 1.276 \\
\hline & & 90 & $5.704^{*}$ & 1.147 & 0.000 & 8.005 & 3.403 \\
\hline & \multirow[t]{3}{*}{60} & 0 & $4.027^{*}$ & 1.124 & 0.001 & 1.772 & 6.282 \\
\hline & & 30 & $3.582^{*}$ & 1.150 & 0.003 & 1.276 & 5.889 \\
\hline & & 90 & $2.122^{*}$ & 1.020 & 0.042 & 4.167 & 0.076 \\
\hline & \multirow[t]{3}{*}{90} & 0 & $6.149^{*}$ & 1.390 & 0.000 & 3.360 & 8.937 \\
\hline & & 30 & $5.704^{*}$ & 1.147 & 0.000 & 3.403 & 8.005 \\
\hline & & 60 & $2.122^{*}$ & 1.020 & 0.042 & 0.076 & 4.167 \\
\hline \multirow{12}{*}{$\begin{array}{l}\text { Flexion moments } \\
(\mathrm{Nmm} / \mathbf{k g})\end{array}$} & \multirow[t]{3}{*}{0} & 30 & 37.741 & 43.019 & 0.384 & 48.545 & 124.027 \\
\hline & & 60 & 98.725 & 55.467 & 0.081 & 12.527 & 209.977 \\
\hline & & 90 & $196.787^{*}$ & 53.372 & 0.001 & 89.736 & 303.838 \\
\hline & \multirow[t]{3}{*}{30} & 0 & 37.741 & 43.019 & 0.384 & 124.027 & 48.545 \\
\hline & & 60 & 60.984 & 45.334 & 0.184 & 29.944 & 151.913 \\
\hline & & 90 & $159.046^{*}$ & 42.688 & 0.000 & 73.424 & 244.668 \\
\hline & \multirow[t]{3}{*}{60} & 0 & 98.725 & 55.467 & 0.081 & 209.977 & 12.527 \\
\hline & & 30 & 60.984 & 45.334 & 0.184 & 151.913 & 29.944 \\
\hline & & 90 & $98.062^{*}$ & 46.029 & 0.038 & 5.740 & 190.384 \\
\hline & \multirow[t]{3}{*}{90} & 0 & $196.787^{*}$ & 53.372 & 0.001 & 303.838 & 89.736 \\
\hline & & 30 & $159.046^{*}$ & 42.688 & 0.000 & 244.668 & 73.424 \\
\hline & & 60 & $98.062^{*}$ & 46.029 & 0.038 & 190.384 & 5.740 \\
\hline \multirow{12}{*}{$\begin{array}{l}\text { Extension moments } \\
(\mathrm{Nmm} / \mathrm{kg})\end{array}$} & \multirow[t]{3}{*}{0} & 30 & $98.843^{*}$ & 33.914 & 0.005 & 30.822 & 166.865 \\
\hline & & 60 & $254.436^{*}$ & 44.212 & 0.000 & 165.759 & 343.114 \\
\hline & & 90 & $323.881^{*}$ & 46.311 & 0.000 & 230.993 & 416.769 \\
\hline & \multirow[t]{3}{*}{30} & 0 & $98.843^{*}$ & 33.914 & 0.005 & 166.865 & 30.822 \\
\hline & & 60 & $155.593^{*}$ & 37.923 & 0.000 & 79.529 & 231.657 \\
\hline & & 90 & $225.037^{*}$ & 46.053 & 0.000 & 132.666 & 317.409 \\
\hline & \multirow[t]{3}{*}{60} & 0 & $254.436^{*}$ & 44.212 & 0.000 & 343.114 & 165.759 \\
\hline & & 30 & $155.593^{*}$ & 37.923 & 0.000 & 231.657 & 79.529 \\
\hline & & 90 & 69.445 & 43.330 & 0.115 & 17.464 & 156.353 \\
\hline & \multirow[t]{3}{*}{90} & 0 & $323.881^{*}$ & 46.311 & 0.000 & 416.769 & 230.993 \\
\hline & & 30 & $225.037^{*}$ & 46.053 & 0.000 & 317.409 & 132.666 \\
\hline & & 60 & 69.445 & 43.330 & 0.115 & 156.353 & 17.464 \\
\hline
\end{tabular}

Continued 
Table 2 Continued

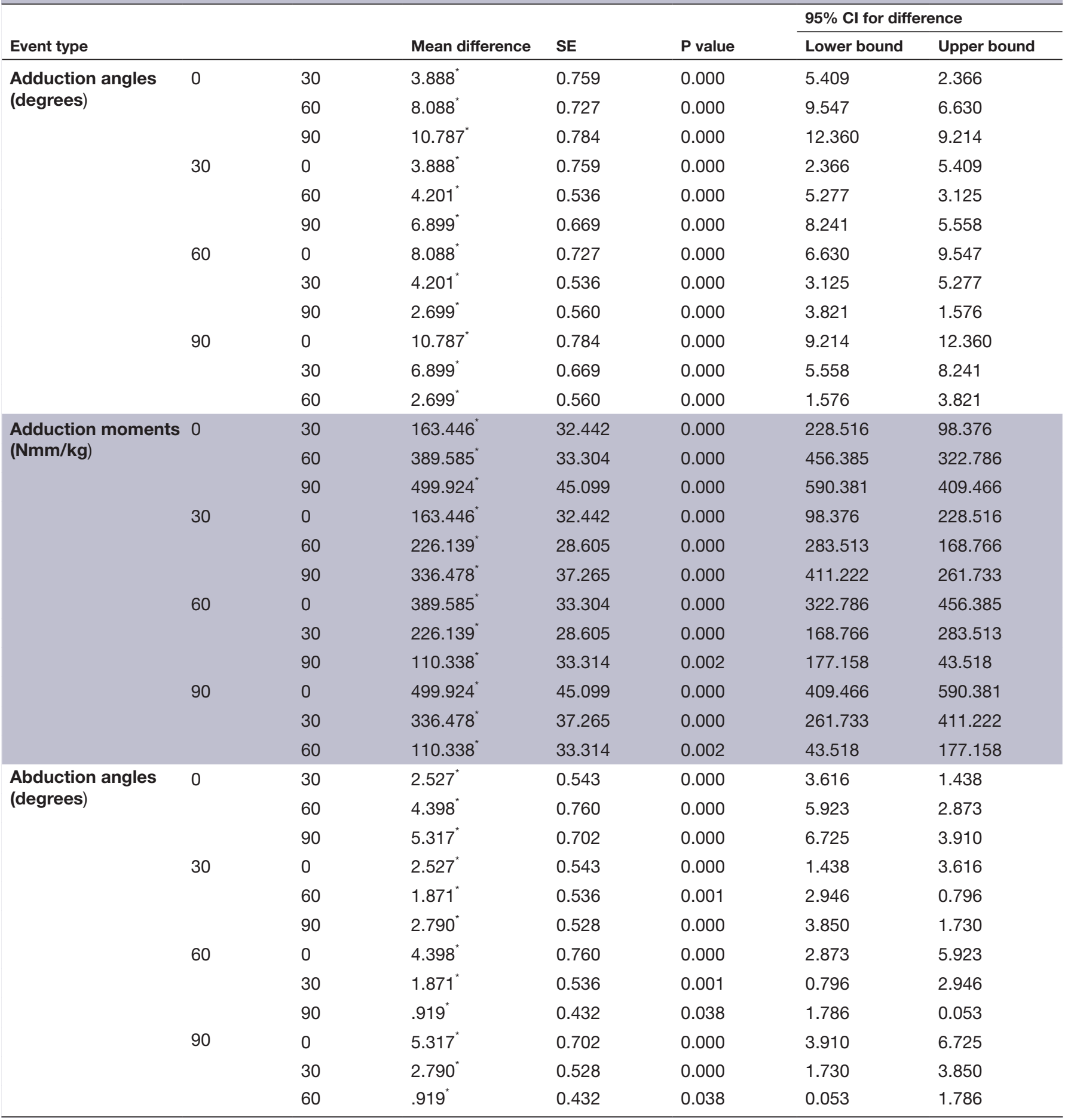




\section{Open access}

Table 2 Continued

\begin{tabular}{|c|c|c|c|c|c|c|c|}
\hline \multirow{2}{*}{ Event type } & & & \multirow[b]{2}{*}{ Mean difference } & \multirow[b]{2}{*}{ SE } & \multirow[b]{2}{*}{$P$ value } & \multicolumn{2}{|c|}{$95 \% \mathrm{Cl}$ for difference } \\
\hline & & & & & & Lower bound & Upper bound \\
\hline \multirow{12}{*}{$\begin{array}{l}\text { Abduction moments } \\
(\mathrm{Nmm} / \mathrm{kg})\end{array}$} & 0 & 30 & $101.129^{*}$ & 34.395 & 0.005 & 170.117 & 32.141 \\
\hline & & 60 & $203.261^{*}$ & 35.802 & 0.000 & 275.071 & 131.450 \\
\hline & & 90 & $329.079^{*}$ & 33.256 & 0.000 & 395.782 & 262.376 \\
\hline & 30 & 0 & $101.129^{*}$ & 34.395 & 0.005 & 32.141 & 170.117 \\
\hline & & 60 & $102.131^{*}$ & 37.945 & 0.009 & 178.240 & 26.023 \\
\hline & & 90 & $227.950^{*}$ & 31.780 & 0.000 & 291.692 & 164.207 \\
\hline & 60 & 0 & $203.261^{*}$ & 35.802 & 0.000 & 131.450 & 275.071 \\
\hline & & 30 & $102.131^{*}$ & 37.945 & 0.009 & 26.023 & 178.240 \\
\hline & & 90 & $125.819^{*}$ & 35.845 & 0.001 & 197.714 & 53.923 \\
\hline & 90 & 0 & $329.079^{*}$ & 33.256 & 0.000 & 262.376 & 395.782 \\
\hline & & 30 & $227.950^{*}$ & 31.780 & 0.000 & 164.207 & 291.692 \\
\hline & & 60 & $125.819^{*}$ & 35.845 & 0.001 & 53.923 & 197.714 \\
\hline \multirow{12}{*}{$\begin{array}{l}\text { Internal rotation } \\
\text { angles (degrees) }\end{array}$} & 0 & 30 & $1.570^{*}$ & 0.547 & 0.006 & 0.473 & 2.667 \\
\hline & & 60 & $2.038^{*}$ & 0.801 & 0.014 & 0.431 & 3.645 \\
\hline & & 90 & $4.184^{*}$ & 0.862 & 0.000 & 2.455 & 5.912 \\
\hline & 30 & 0 & $1.570^{*}$ & 0.547 & 0.006 & 2.667 & 0.473 \\
\hline & & 60 & 0.468 & 0.595 & 0.435 & 0.726 & 1.662 \\
\hline & & 90 & $2.613^{*}$ & 0.694 & 0.000 & 1.222 & 4.005 \\
\hline & 60 & 0 & $2.038^{*}$ & 0.801 & 0.014 & 3.645 & 0.431 \\
\hline & & 30 & 0.468 & 0.595 & 0.435 & 1.662 & 0.726 \\
\hline & & 90 & $2.146^{*}$ & 0.639 & 0.001 & 0.864 & 3.427 \\
\hline & 90 & 0 & $4.184^{*}$ & 0.862 & 0.000 & 5.912 & 2.455 \\
\hline & & 30 & $2.613^{*}$ & 0.694 & 0.000 & 4.005 & 1.222 \\
\hline & & 60 & $2.146^{*}$ & 0.639 & 0.001 & 3.427 & 0.864 \\
\hline \multirow{12}{*}{$\begin{array}{l}\text { Internal rotation } \\
\text { moments }(\mathrm{Nmm} / \mathbf{k g})\end{array}$} & 0 & 30 & 17.640 & 9.194 & 0.060 & 36.081 & 0.800 \\
\hline & & 60 & $61.764^{*}$ & 10.622 & 0.000 & 83.068 & 40.459 \\
\hline & & 90 & $71.546^{*}$ & 11.499 & 0.000 & 94.610 & 48.482 \\
\hline & 30 & 0 & 17.640 & 9.194 & 0.060 & 0.800 & 36.081 \\
\hline & & 60 & $44.123^{*}$ & 10.839 & 0.000 & 65.863 & 22.384 \\
\hline & & 90 & $53.906^{*}$ & 11.606 & 0.000 & 77.185 & 30.626 \\
\hline & 60 & 0 & $61.764^{*}$ & 10.622 & 0.000 & 40.459 & 83.068 \\
\hline & & 30 & $44.123^{*}$ & 10.839 & 0.000 & 22.384 & 65.863 \\
\hline & & 90 & 9.783 & 12.459 & 0.436 & 34.773 & 15.207 \\
\hline & 90 & 0 & $71.546^{*}$ & 11.499 & 0.000 & 48.482 & 94.610 \\
\hline & & 30 & $53.906^{*}$ & 11.606 & 0.000 & 30.626 & 77.185 \\
\hline & & 60 & 9.783 & 12.459 & 0.436 & 15.207 & 34.773 \\
\hline
\end{tabular}

Continued 
Table 2 Continued

\begin{tabular}{|c|c|c|c|c|c|c|c|}
\hline \multirow{2}{*}{ Event type } & & & \multirow[b]{2}{*}{ Mean difference } & \multirow[b]{2}{*}{ SE } & \multirow[b]{2}{*}{$P$ value } & \multicolumn{2}{|c|}{$95 \% \mathrm{Cl}$ for difference } \\
\hline & & & & & & Lower bound & Upper bound \\
\hline \multirow{12}{*}{$\begin{array}{l}\text { External rotation } \\
\text { angles (degrees) }\end{array}$} & 0 & 30 & $4.151^{*}$ & 1.243 & 0.002 & 1.658 & 6.644 \\
\hline & & 60 & $8.640^{*}$ & 1.222 & 0.000 & 6.188 & 11.091 \\
\hline & & 90 & $10.109^{*}$ & 1.295 & 0.000 & 7.511 & 12.707 \\
\hline & 30 & 0 & $4.151^{*}$ & 1.243 & 0.002 & 6.644 & 1.658 \\
\hline & & 60 & $4.489^{*}$ & 1.121 & 0.000 & 2.239 & 6.738 \\
\hline & & 90 & $5.958^{*}$ & 1.209 & 0.000 & 3.534 & 8.383 \\
\hline & 60 & 0 & $8.640^{*}$ & 1.222 & 0.000 & 11.091 & 6.188 \\
\hline & & 30 & $4.489^{*}$ & 1.121 & 0.000 & 6.738 & 2.239 \\
\hline & & 90 & 1.470 & 1.116 & 0.194 & 0.769 & 3.709 \\
\hline & 90 & 0 & $10.109^{*}$ & 1.295 & 0.000 & 12.707 & 7.511 \\
\hline & & 30 & $5.958^{*}$ & 1.209 & 0.000 & 8.383 & 3.534 \\
\hline & & 60 & 1.470 & 1.116 & 0.194 & 3.709 & 0.769 \\
\hline \multirow{12}{*}{$\begin{array}{l}\text { External rotation } \\
\text { moments }(\mathrm{Nmm} / \mathrm{kg})\end{array}$} & 0 & 30 & 1.964 & 14.036 & 0.889 & -26.188 & 30.117 \\
\hline & & 60 & -12.391 & 15.724 & 0.434 & -43.930 & 19.148 \\
\hline & & 90 & -15.810 & 12.942 & 0.227 & -41.769 & 10.149 \\
\hline & 30 & 0 & -1.964 & 14.036 & 0.889 & -30.117 & 26.188 \\
\hline & & 60 & -14.355 & 12.183 & 0.244 & -38.791 & 10.080 \\
\hline & & 90 & -17.774 & 11.806 & 0.138 & -41.455 & 5.907 \\
\hline & 60 & 0 & 12.391 & 15.724 & 0.434 & -19.148 & 43.930 \\
\hline & & 30 & 14.355 & 12.183 & 0.244 & -10.080 & 38.791 \\
\hline & & 90 & -3.419 & 12.410 & 0.784 & -28.311 & 21.473 \\
\hline & 90 & 0 & 15.810 & 12.942 & 0.227 & -10.149 & 41.769 \\
\hline & & 30 & 17.774 & 11.806 & 0.138 & -5.907 & 41.455 \\
\hline & & 60 & 3.419 & 12.410 & 0.784 & -21.473 & 28.311 \\
\hline
\end{tabular}

*statistically significant $(p<0.05)$

\section{Knee}

Flexion

Angles

For flexion at the knee, $0^{\circ}$ and $30^{\circ}$ produced the lowest angulation (44.6 $6^{\circ}$ of angulation for $0^{\circ}$ foot position), and there was no significant difference between them. The highest mean flexion was recorded from $90^{\circ}$ with a mean angle of $50.7^{\circ}$. Very significant differences were found between $90^{\circ}$ with both $0^{\circ}$ and $30^{\circ}$ and also with $60^{\circ}$ and $0^{\circ}(\mathrm{p}<0.001)$. Significant differences were also found between $60^{\circ}$ and both $30^{\circ}$ and $90^{\circ}(\mathrm{p}<0.05)$.

\section{Moments}

For flexion at the knee, a foot position of $90^{\circ}$ produced the lowest maximum flexion moments with a mean of $1282 \mathrm{Nmm} / \mathrm{kg}$, while $0^{\circ}$ produced the highest with a mean of $1479 \mathrm{Nmm} / \mathrm{kg}$. The result for $90^{\circ}$ was significantly different to that of $60^{\circ} \quad(\mathrm{p}<0.05)$ and of greater significant difference to $0^{\circ}$ and $30^{\circ}(\mathrm{p}<0.001)$.

\section{Extension}

Angles

No knee extension angles were recorded.

\section{Moments}

For extension at the knee, a foot position of $90^{\circ}$ produced the highest maximum extension moments, with a mean of $591 \mathrm{Nmm} / \mathrm{kg}$. This result was significantly different from the maximum moments produced at foot positions of both $0^{\circ}$ and $30^{\circ}$, of which the mean extension moments were $267 \mathrm{Nmm} / \mathrm{kg}$ and $366 \mathrm{Nmm} / \mathrm{kg}$, respectively $(\mathrm{p}<0.001)$.

\section{Adduction}

\section{Angles}

Very significant differences were found between adduction angles of all foot positions $(p<0.001)$, the lowest resulting from $0^{\circ}$ and the highest from $90^{\circ}$.

\section{Moments}

A foot position of $0^{\circ}$ produced the lowest adduction moments $(683 \mathrm{Nmm} / \mathrm{kg})$ and $90^{\circ}$ produced the highest $(1183 \mathrm{Nmm} / \mathrm{kg})$. A significant difference was found between $60^{\circ}$ and $90^{\circ}(\mathrm{p}<0.05)$ and greater significant differences were found between all other foot positions $(\mathrm{p}<0.001)$. 


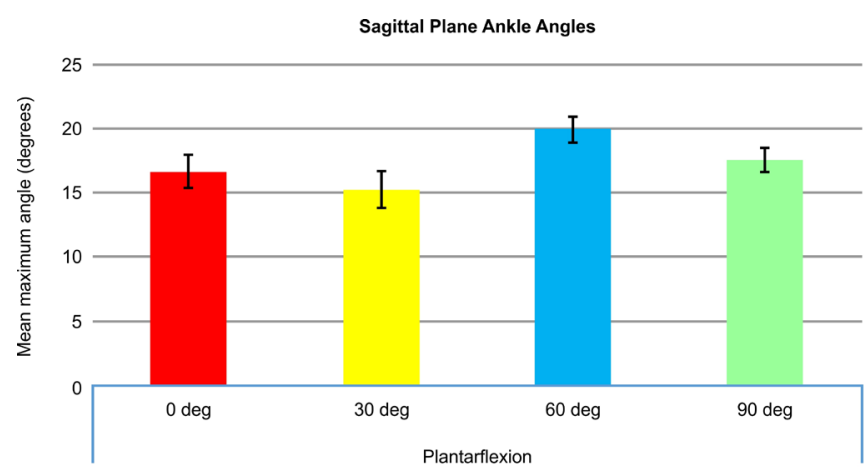

Coronal Plane Ankle Angles

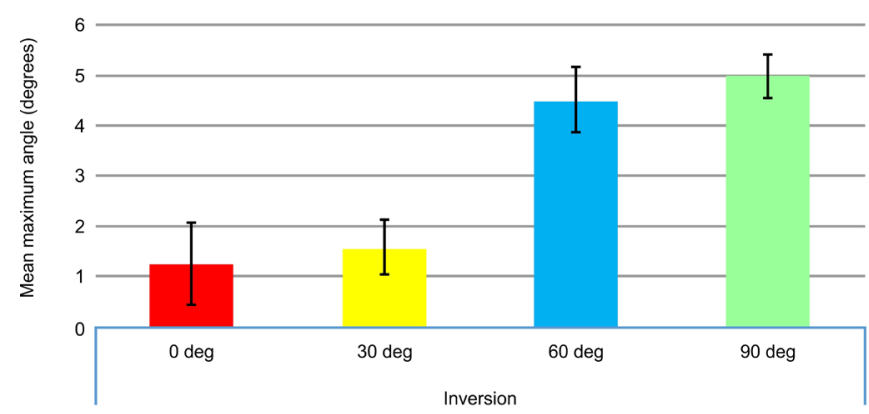

Transverse Plane Ankle Angles

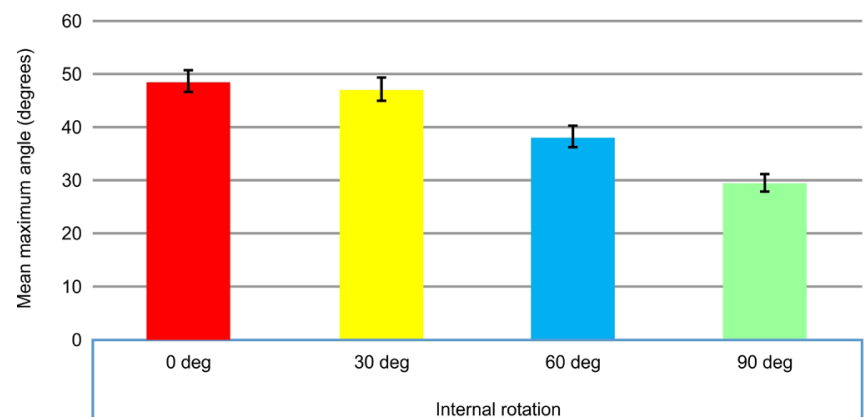

Figure 3 Ankle graphs.

Abduction

\section{Angles}

For abduction, $90^{\circ}$ produced the lowest angulation of $6.3^{\circ}$ and $0^{\circ}$ produced the highest of $11.6^{\circ}$. A significant difference was found between $60^{\circ}$ and $90^{\circ}(\mathrm{p}<0.05)$ and very significant differences were found between all other foot positions $(p<0.001)$.

\section{Moments}

For abduction, the trend followed the opposite direction, with the highest moments at $0^{\circ}(539 \mathrm{Nmm} / \mathrm{kg})$. Significant differences were found between $30^{\circ}$ and both $0^{\circ}$ and $60^{\circ}(\mathrm{p}<0.05)$. Even greater significant differences were found between $60^{\circ}$ and both $0^{\circ}$ and $90^{\circ}$, and between $90^{\circ}$ and both $0^{\circ}$ and $30^{\circ}(\mathrm{p}<0.001)$.
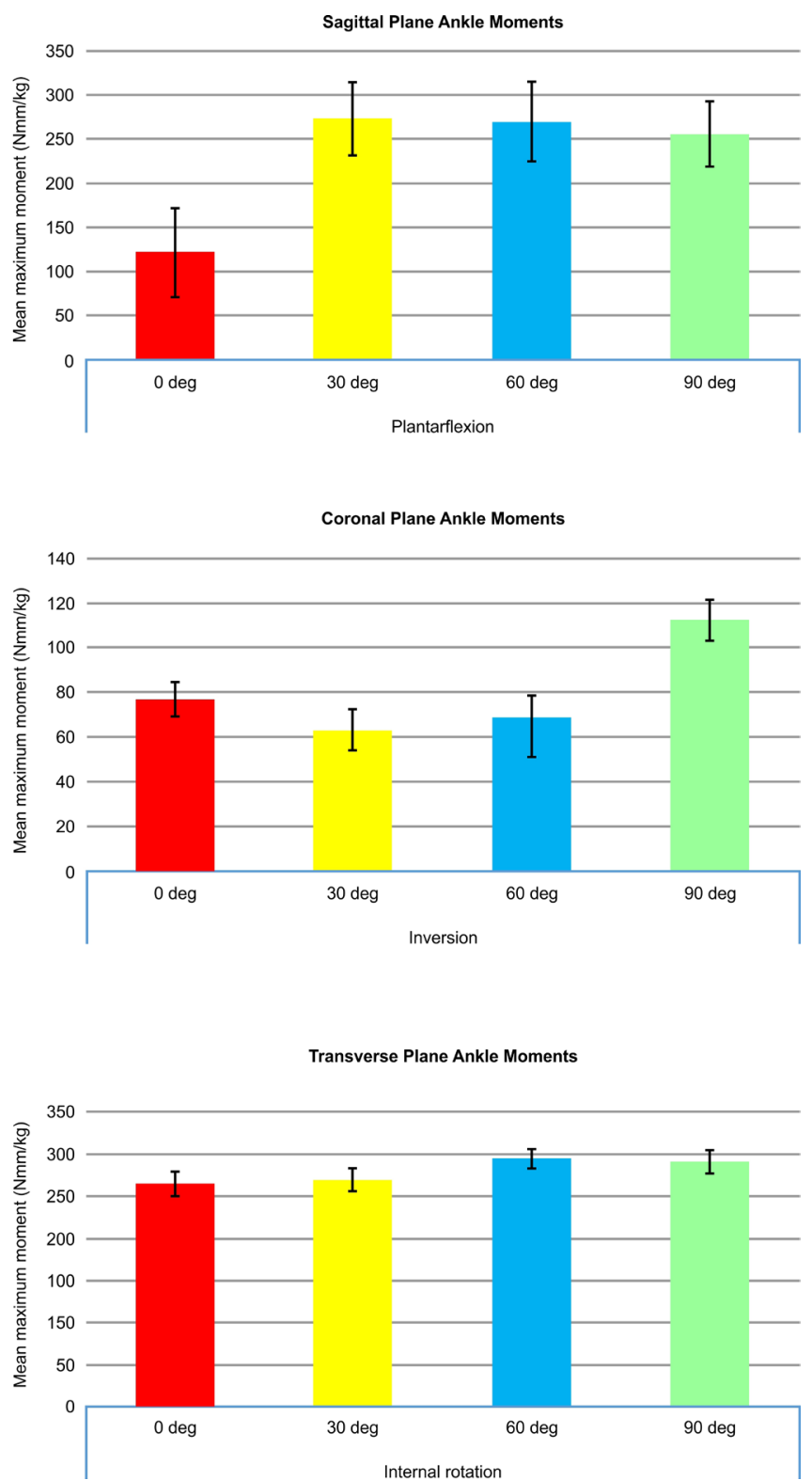

Internal rotation

Angles

Figure 4 displays a trend of decreasing internal rotation angles from $0^{\circ}$ to $90^{\circ}$. For internal rotation angles, very significant differences were found between $90^{\circ}$ and both $0^{\circ}$ and $30^{\circ}(\mathrm{p}<0.001)$. Significant differences were also found between $0^{\circ}$ and $30^{\circ}$ and also $60^{\circ}$ with both $0^{\circ}$ and $90^{\circ}(\mathrm{p}<0.05)$.

\section{Moments}

The foot positions that produced the lowest internal rotation moments were $0^{\circ}$ and $30^{\circ}$, with no significant difference between them. The lowest was $0^{\circ}$ with a mean moment of $202 \mathrm{Nmm} / \mathrm{kg}$. The highest internal rotation moments were produced at $60^{\circ}$ and $90^{\circ}$, with no significant difference between them. The highest was $90^{\circ}$ with a mean moment of $274 \mathrm{Nmm} / \mathrm{kg}$. Significant differences 
Sagittal Plane Knee Angles

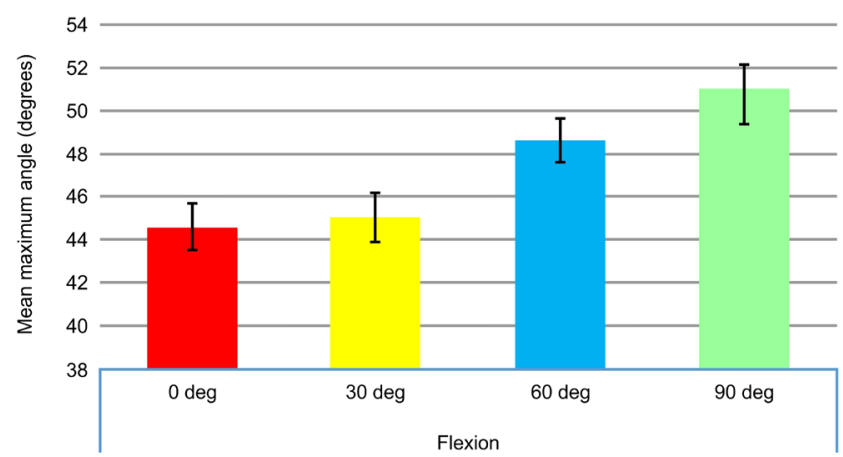

Coronal Plane Knee Angles

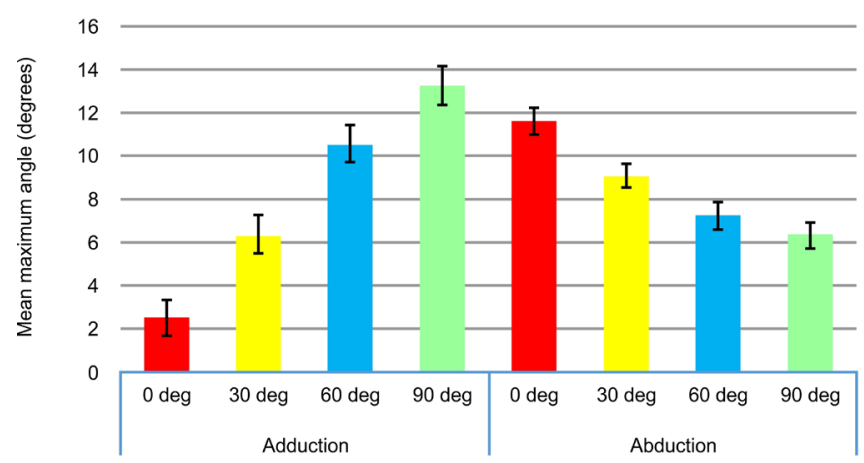

Transverse Plane Knee Angles

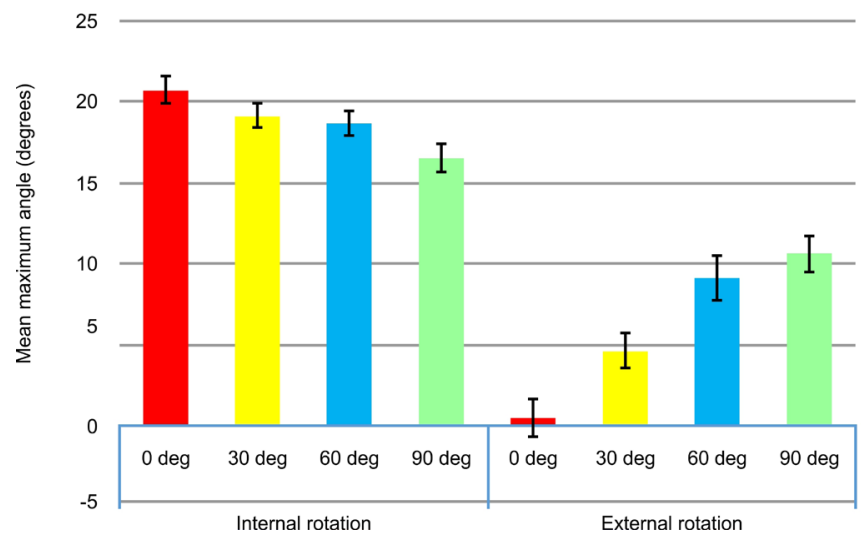

Figure 4 Knee graphs.

were found between both $0^{\circ}$ and $30^{\circ}$ with both $60^{\circ}$ and $90^{\circ}(\mathrm{p}<0.001)$.

\section{External rotation}

\section{Angles}

The lowest external rotation angle was found to be at $0^{\circ}$ with a mean of $0.5^{\circ}$, and the highest angle was found at $90^{\circ}$ with a mean of $10.6^{\circ}$. A significant difference was found between $0^{\circ}$ and $30^{\circ} \quad(p<0.05)$. Very significant differences were found between all other foot positions $(p<0.001)$, except between $60^{\circ}$ and $90^{\circ}$, where there was no significant difference.
Sagittal Plane Knee Moments

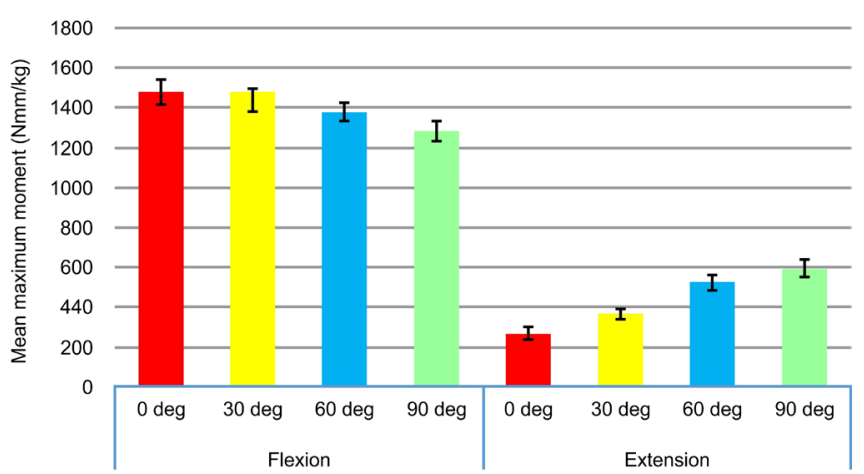

Coronal Plane Knee Moments

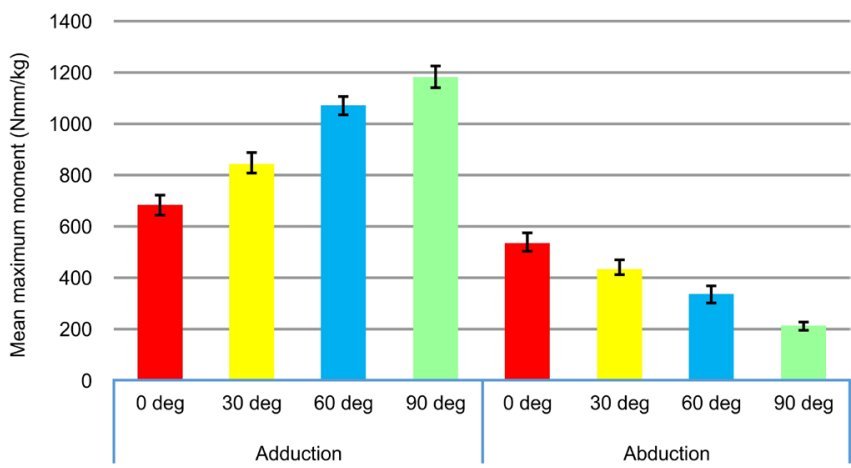

Transverse Plane Knee Moments

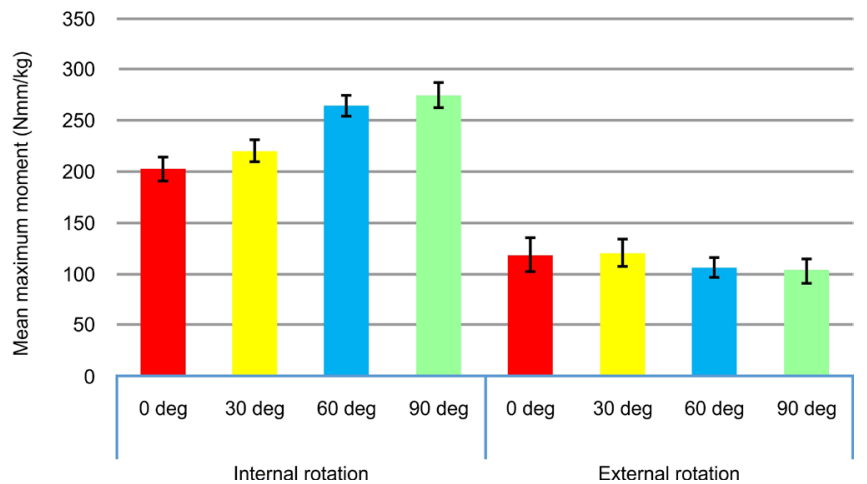

Moments

No significant differences were found for external rotation moments between any of the four foot positions.

\section{DISCUSSION}

This study investigated which foot position $\left(0^{\circ}, 30^{\circ}, 60^{\circ}\right.$ or $90^{\circ}$ ) produced the smallest and largest degrees of angulation and moments, in the lead ankle and knee joints, during the hockey hit.

\section{Ankle summary}

Ankle injury in hockey most commonly involves the lateral ligaments ${ }^{3}$ which usually occurs when the foot 
is inverted, internally rotated and plantarflexed. ${ }^{5}$ The highest degrees of ankle inversion were found at foot positions of $0^{\circ}$ and $90^{\circ}$ and that of internal rotation was found at a foot position of $0^{\circ}$. Although $0^{\circ}$ consistently produced the highest angulation, $90^{\circ}$ caused the most significantly high moments. Therefore, rather than one particular foot position, it seems that the extremes of foot position collectively lead to larger degrees of angulation and magnitudes of moment. In contrast, $30^{\circ}$ was the foot position that most consistently produced the lowest degrees of angulation and moments.

\section{Knee summary}

For the knee, moments in the coronal plane were significantly higher at foot positions of $0^{\circ}$ and $90^{\circ}$ compared with $30^{\circ}(\mathrm{p}<0.001)$, and moments in the transverse plane were significantly higher at both $60^{\circ}$ and $90^{\circ}$ than $0^{\circ}$ and $30^{\circ}(\mathrm{p}<0.001)$.

\section{Limitations}

This study is an exploratory study to aid future research and hence the relatively low number of participants. While the surface on which the hit was performed did not replicate normal playing conditions, the key focus of the study was to propose the best foot position from the four positions investigated. As such, the surface was constant throughout the study, hence the four foot positions could be directly compared against one another. Furthermore, the proposed foot position of $30^{\circ}$ may not be the most appropriate for all hockey players and it is not expected that a player would be able to consistently implement this into their play. However, alignment of the lower limb could become a more prominent aspect of hockey coaching and could be of particular relevance to players with a history of injury.

\section{CONCLUSION}

The aim of this study was fulfilled, indicating that lead foot position is related to the angles and moments produced in the lower limb joints during the hockey hit, and the null hypothesis can, therefore, be rejected. A lead foot position of $30^{\circ}$ resulted in the smallest degrees of angulation, and magnitude of moment, the most often, and the largest the least often. This correlates to a lead foot that is in line with the rest of the body, while carrying out the hockey hit. The idea that this may be correlated with injury risk would require testing via either an intervention or epidemiological study, and if this idea was confirmed, a specific intervention associated with foot position during the hockey hit may decrease the risk of injury.
Acknowledgements The authors would like to thank lan Christie for his valuable assistance in the production of bespoke images.

Contributors FEF: planning the study, conducting the study, analysing the data, reporting the study, generating the draft write-up-responsible for overall content as guarantor. GPA: data collection for study and Vicon markers repeatability. SN: data collection for study and Vicon software reliability. WWW: statistical analysis of data. RA: reporting the study, revising the original and revision manuscript critically for intellectual content, submitting the study—responsible for overall content as guarantor.

Funding The study was funded internally by the department.

Competing interests None declared.

Patient consent for publication Not required.

Ethics approval The study was approved by the Medical School Research Ethics Committee-ID: SMED REC 069/17.

Provenance and peer review Not commissioned; externally peer reviewed.

Data availability statement № data are available.

Open access This is an open access article distributed in accordance with the Creative Commons Attribution Non Commercial (CC BY-NC 4.0) license, which permits others to distribute, remix, adapt, build upon this work non-commercially, and license their derivative works on different terms, provided the original work is properly cited, appropriate credit is given, any changes made indicated, and the use is non-commercial. See: http://creativecommons.org/licenses/by-nc/4.0/.

\section{ORCID iD}

Rami Abboud http://orcid.org/0000-0002-1753-9606

\section{REFERENCES}

1 Theilen T-M, Mueller-Eising W, Wefers Bettink P, et al. Injury data of major international field hockey tournaments. Br J Sports Med 2016;50:657-60.

2 Johnston T, Brown S, Kaliarntas K, et al. Non-Contact injury incidence and warm up observation in hockey in Scotland. Br J Sports Med 2016;50:e4.10-e4.

3 Barboza SD, van Mechelen W, Verhagen E. Monitoring field hockey injuries: the first step for prevention. Br J Sports Med 2017;51:312.1-312.

4 Ncaa.org. The National collegiate athletic association, 2009. Available: https://www.ncaa.org/sites/default/files/NCAA FieldHockey_Injuries_HiRes.pdf [Accessed 6 Jan 2018].

5 Kerr R, Arnold GP, Drew TS, et al. Shoes influence lower limb muscle activity and may predispose the wearer to lateral ankle ligament injury. Journal of Orthopaedic Research 2009;27:318-24.

6 Ramanathan AK, Parish EJ, Arnold GP, et al. The influence of shoe sole's varying thickness on lower limb muscle activity. Foot and Ankle Surgery 2011;17:218-23.

7 Ramanathan AK, Wallace DT, Arnold GP, et al. The effect of varying footwear configurations on the peroneus longus muscle function following inversion. Foot 2011;21:31-6.

8 Brockett CL, Chapman GJ. Biomechanics of the ankle. Orthop Trauma 2016;30:232-8.

9 Wild C, Rosalie S, Sherry D, et al. The relationship between front foot position and lower limb and lumbar kinetics during a DraG flick in specialist hockey players. J Sci Med Sport 2017;20:e26.

$10 \mathrm{Kim} \mathrm{T}$, Kim E, Choi H. Effects of a 6-week neuromuscular rehabilitation program on Ankle-Evertor strength and postural stability in elite women field hockey players with chronic ankle instability. J Sport Rehabil 2017;26:269-80.

11 Christie IS. Force_plates_plan_view.jpg. [Art] (Department of Orthopaedic and Trauma Surgery, University of Dundee) 2018.

12 Christie IS. Lower_limb_marker_placement.jpg [custom image] ( ). [Art] (Department of Orthopaedic and Trauma Surgery, University of Dundee) 2018. 